\title{
In-Situ Super Rapid Heating of Nano-Aluminum and Nano-Thermites
}

\author{
Kyle S. Sullivan ${ }^{1}$, Wen-An Chiou ${ }^{2}$, Richard Fiore ${ }^{3}$ and Michael Zachariah ${ }^{1}$ \\ 1. Department of Mechanical Engineering, University of Maryland, College Park, MD 20742 \\ 2. NISP Lab, NanoCenter, University of Maryland, College Park, MD 20742-2831 \\ 3. Protochips, Inc., 617 Hutton St, Suite 111, Raleigh, NC 27606
}

Thermites are energetic systems comprised of a metal fuel and a metal/metal oxide oxidizer, which give rise to high temperatures and self-propagating reactions upon ignition. When nanoparticles are used in place of larger particles, more homogeneous mixing and interfacial contact can be achieved, leading to several orders of magnitude increase in combustion characteristics[1]. Nano-Al is traditionally used as a fuel due to its high energy density and low cost, and several oxidizers have been studied. These materials are currently being investigated for several applications in propellants, pyrotechnics and explosives, where controlled energy release is a desirable trait. Nano-Al naturally forms oxide shell that is several nanometers in thickness. The interaction of the $\mathrm{Al}$ core (MP $933{ }^{\circ} \mathrm{K}$ ) with the $\mathrm{Al}$ oxide shell $\left(\mathrm{MP} 2327{ }^{\circ} \mathrm{K}\right)$ is critical to understanding the initiation mechanism. Two different hypotheses have prevailed $[2,3,4]$ as to the behavior of nano-Al subjected to rapid heating. However, resolving this issue requires experimental support, which is currently lacking. This research examines the initiation mechanism by replicating a super rapid heating environment in an electron microscope. Both nano- $\mathrm{Al}$ and a nano- $\mathrm{Al} / \mathrm{WO}_{3}$ thermite were investigated.

The nano-Al, designated as "50nm ALEX" (70\% elemental by mass as measured by thermogravimetric analysis), was purchased from the Argonide Corp. The $\mathrm{WO}_{3}$ with a size of $<100 \mathrm{~nm}$ (as specified by the supplier) was purchased from Sigma Aldrich. The thermite was prepared by weighing stoichiometric amounts of nano- $\mathrm{Al}$ and $\mathrm{WO}_{3}$ powders and adding into a $5 \mathrm{ml}$ vial with some hexane. It was then sonicated for $\sim 30 \mathrm{~min}$. to ensure intimate mixing. A few drops of ethanol was then added after the evaporation of hexane, and the suspended sample was pipetted onto a special semiconductor-based heating grid/stage (Protochips, Inc.) that enabled heating of a sample from room temperature up to a maximum of $1575^{\circ} \mathrm{K}$ at a rate of $10^{6} \mathrm{~K} / \mathrm{s}$. Selected areas of the same particles were examined before and after heating.

Initial TEM heating study of nano-Al depicted a morphological change of many of the particles after one millisecond (1 ms) heating (Fig. 1). No "spallation" or ejection of molten Al was observed, contrary to the prediction of ref. 2. The result is consistent with a cracking/diffusion initiation mechanism for nano-Al. BSE images and EDS mapping of the untreated thernite sample reveal the random distribution of $\mathrm{Al}$ and $\mathrm{WO}_{3}$ particles (Fig. 2). Al particles tended to be spherical and ranged from 50 to $100 \mathrm{~nm}$ with some up to $800 \mathrm{~nm}$, whereas $\mathrm{WO}_{3}$ particles ranged from 100 to $300 \mathrm{~nm}$ in general and appeared more euhedral in shape. Larger $\mathrm{Al}$ particles were more or less surrounded by $\mathrm{WO}_{3}$ particles. The BSE image shows that the surface of $\mathrm{Al}$ particles appeared relatively clean, though some very small particles seem to be ahered on the surface. The majority of these particles changed instantly after $1 \mathrm{~ms}$ of heating to $1573{ }^{\circ} \mathrm{K}$. Most noticeably, Al particles appeared flattened and coated with a thin layer of $\mathrm{W}$ and/or $\mathrm{WO}_{3}$ (Fig. 3). The BSE image and EDS mapping clearly depicted the distribution of high atomic number W from Al. Possible evidence of elemental interdifussion was observed in the high resolution SEM/EDS images and EDS data. This finding supports heterogeneous condensed-phase reactions as part of the initiation mechanism in the nano- $\mathrm{Al} / \mathrm{WO}_{3}$ thermite. With further additional heating of $1 \mathrm{~ms}$, an even more drastic change was observed (Fig. 4). The small bright spots, observed in the BSE image, may indicate the formation of solid $\mathrm{W}$ particles, evidence of further extent of reaction. Other thermite systems are currently being investigated.

1. C. E. Aumann et al., J. Vac. Sci. \& Tech. B 13 (3) (1995) 1178.

2. V. I. Levitas et al., J. App. Phys. 101 (2007) 083524.

3. A. Rai et al., Comb. Theory and Mod. 10 (2006) 843.

4. M. A. Trunov et al., Comb. Theory and Mod. 10 (2006) 603.

5. This work was supported by the Army Research Office and the NISP Lab (MRSEC Shared Facility, NSF DMR 05-20741) at the University of Maryland. Special thanks to Dr. L. Lai for technical assistance. 


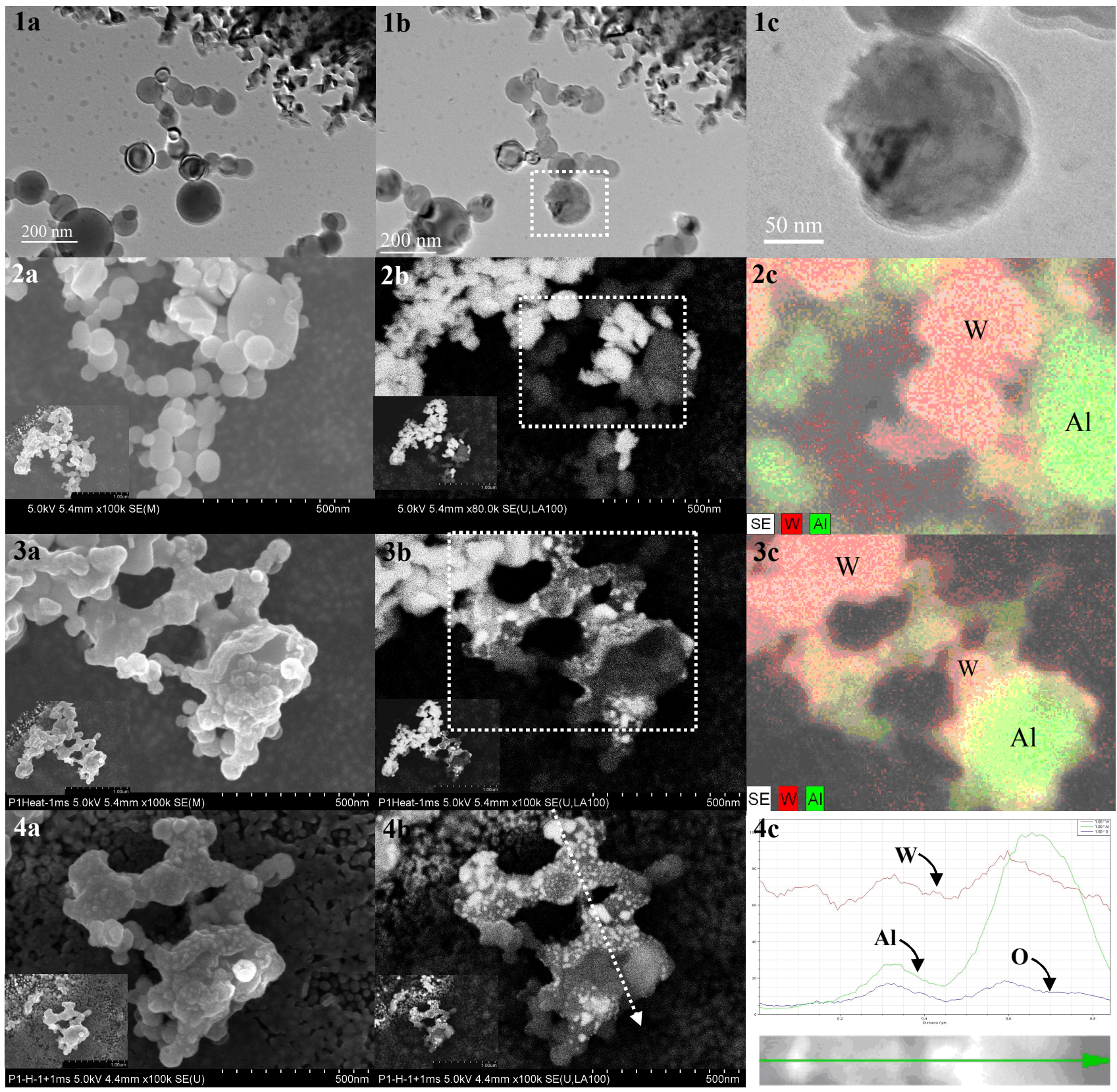

Fig. 1. TEM images of nano-Al particles before (a) and after (b) rapid heating to $1573{ }^{\circ} \mathrm{K}$ in $1 \mathrm{~ms}$. High magnification micrograph (c) of the boxed area (in b) illustrates a change in the morphology, indicating a breakdown/cracking of the oxide shell. No "spallation" or ejection of Al was observed.

Fig. 2. Fig. 1. SEM image (a) shows $\mathrm{Al}$ and $\mathrm{WO}_{3}$ particles. BSE image (b) (bright: $\mathrm{WO}_{3}$; lighter contrast: $\mathrm{Al}$ ) and EDS mapping (c) of $\mathrm{W}$ and $\mathrm{Al}$, confirms the random distribution of these two elemental nanoparticles. Inserts reveal low magnification view. EDS mapping was obtained from the boxed area.

Fig. 3. SEM (a) and BSE (b) images and EDS mapping (c) show the morphological change after $1 \mathrm{~ms}$ heating to $1573{ }^{\circ} \mathrm{K}$. It appears that $\mathrm{W} / \mathrm{WO}_{3}$ has coated the $\mathrm{Al}$ surface.

Fig. 4. SEM (a) and BSE (b) images depict further morphological change, especially volumetric change and particle redistribution after an additional $1 \mathrm{~ms}$ heating $(2 \mathrm{~ms}$ in total). The EDS line profile (dash line on the BSE image) shows possible interdiffusion of $\mathrm{Al} / \mathrm{WO}_{3}$. Note the appearance of small bright spots/particles in the BSE images. 